\title{
Metodología para el balanceo de rotores empleando un analizador de vibraciones
}

\section{Methodology for rotors balancing using a vibration analyzer}

\author{
Heller Sánchez-Acevedo ${ }^{1}$, Fabian R. Nova² ${ }^{2}$ Jhon A. Madrigal $^{3}$ \\ ${ }^{1}$ Grupo de Investigación en Energía y Medio Ambiente (Giema), Escuela de Ingeniería Mecánica, Universidad Industrial de \\ Santander, Colombia. Orcid: 0000-0003-0081-2212. Email: gsanche@uis.edu.co \\ ${ }^{2}$ Escuela de Ingeniería Mecánica, Universidad Industrial de Santander, Colombia. Email: fabian_0930@ hotmail.com \\ ${ }^{3}$ Escuela de Ingeniería Mecánica, Universidad Industrial de Santander, Colombia. Email: jalexandermadrigal@gmail.com
}

Recibido: marzo 2, 2018. Aceptado: abril 14, 2018. Versión final: junio 3, 2018.

\begin{abstract}
Resumen
El presente trabajo se centra en el planteamiento de una metodología para el balanceo de rotores. Para la consecución de este objetivo se emplea un analizador de vibraciones, de referencia OROS 35, y sus aplicaciones y acelerómetros. Los diferentes tipos de desbalanceo son revisados y evaluados en este trabajo. La metodología es implementada y ejecutada de forma experimental, en diferentes bancos de ensayos de vibraciones, con diferentes configuraciones. De igual forma, la metodología fue usada con una máquina industrial. Los resultados obtenidos fueron positivos. Estos fueron comparados con la normatividad vigente.
\end{abstract}

Palabras clave: desbalanceo; rotores; vibraciones de máquinas.

\begin{abstract}
The present work focuses on the proposal of a methodology for rotors balancing. To achieve the objective, the vibration analyzer, reference OROS 35 and its applications, are used. The different types of unbalances are reviewed and evaluated at work. The methodology is implemented and executed experimentally in different vibration test benches, with different settings. In the same way, the methodology was used with an industrial machine. The results obtained were positive. These results were then compared with the current regulation.
\end{abstract}

Keywords: mechanical vibration; rotors; unbalance.

\section{Introducción}

La alta competencia industrial y la tecnificación de los procesos industriales han generado un creciente desarrollo tecnológico enfocado en las prácticas de mantenimiento, principalmente en el predictivo. En la maquinaria rotativa, el control sobre las fuerzas dinámicas, principales generadores de las grandes amplitudes de vibración y, por tanto, de los problemas de fatiga y desgaste, ha sido el foco de estudio de cara a evitar la consumación de fallas catastróficas [1].
Los métodos empleados para el monitoreo y establecimiento de la condición de la operación de las máquinas también se han ido desarrollando, incluido el análisis de vibraciones mecánicas, consolidada como una de las técnicas no destructivas más empleadas y versátiles $[2][3][4][5]$.

Una de las fuentes más comunes de vibración en máquinas rotativas es el desbalanceo [6][7]. Por lo tanto, el balanceo de máquinas y equipos industriales, como turbinas y ventiladores, entre otros, se considera 
fundamental en los procesos de fabricación y mantenimiento industrial [8][9][10].

Actualmente existen diferentes técnicas empleadas para reducir el desbalanceo en máquinas rotativas. Como el empleado por Zhou y Shi [11] y Foiles [12], quienes han postulado diversos métodos para balanceo de rotores, aplicando técnicas holoespectrales [13], y métodos de optimización con algoritmos genéticos para reducir las vibraciones del desbalanceo [14]. Otros desarrollos para balancear apuntan al empleo de instrumentos virtuales, soportes del proceso, basados en el método de coeficientes de influencia [1], e incluso técnicas para balanceo de rotores acoplados directamente a un motor de inducción utilizando la corriente residual [15], o empleando el método modal [16]. También existe el balanceo en equipos de alta precisión, sistemas en los que la más mínima fuerza centrífuga afecta de forma importante su condición de operación, como es el caso de los satélites [17] y las herramientas de corte [18][19].

Es así como el presente trabajo se enfoca en reportar el procedimiento para el balanceo dinámico de ejes, fundamentado en la medida de vibraciones en los apoyos, empleando un analizador de señales y una aplicación para realizar los cálculos respectivos. El procedimiento está proyectado para ser aplicado en el sitio de operación del eje que se va a balancear, y evitar el desmontaje del eje y el uso de una máquina balanceadora dinámica.

\section{Desbalanceo}

El desbalanceo se puede definir como una medida que cuantifica la distancia a la que se desplaza la línea central de masa del rotor de la línea central de rotación [16]. La fuerza generada por el desbalanceo, la fuerza centrífuga, se calcula en función de la masa de desbalanceo $(m)$, la excentricidad $(r)$ y la velocidad de rotación $(\omega)$, según la ecuación (1).

$$
F_{\text {centrifuga }}=m *\left(r * \omega^{2}\right)
$$

No obstante, hay que recordar que, en función del tipo de rotor, su dinámica puede ser mucho más compleja, y el cálculo de las aceleraciones también.

El desbalanceo se presenta de forma sincrónica a la velocidad de rotación de la máquina, y su dirección es fundamentalmente radial. Por lo tanto, en el espectro de vibraciones las amplitudes de vibración asociadas a este fallo se registran en la componente $1 \mathrm{X}$ (primer armónico) [20].

Respecto de los tipos de desbalanceo, la norma ISO 1925 los clasifica, según la distribución de masas desbalanceadoras, en desbalanceo estático, cuando el eje principal de inercia es paralelo al eje de rotación; desbalanceo por fuerzas par, cuando el eje principal de inercia intercepta al eje de rotación en el centro de gravedad del rotor; desbalanceo cuasiestático, cuando el eje principal de inercia intercepta al eje de rotación en un punto distinto al centro de gravedad del rotor, y desbalanceo dinámico, cuando el eje principal de inercia no intercepta al eje de rotación.

A partir de la geometría, la distribución de masas y la velocidad de operación del rotor, el balanceo se puede realizar corrigiendo en un plano (balanceo estático), y en dos o más planos (balanceo dinámico) [1]. Dada la norma ISO 5406, se obtienen los modelos para balanceo teniendo en cuenta la relación entre el largo y el diámetro del rotor. En la figura 1, se exponen estos modelos. En la norma ISO 1940 se establecen los niveles máximos de desbalanceo permitidos para las diferentes aplicaciones industriales.

\section{Planteamiento del procedimiento}

El procedimiento planteado inicia con la configuración de la aplicación a través de la que, por una parte, se definen todos los parámetros requeridos para la ejecución del balanceo y, por otra, los cálculos y orientaciones para la realización del proceso correctivo. El analizador empleado es un OROS 35 y sus aplicaciones correspondientes.

\subsection{Configuración de la aplicación}

Configuración inicial. Aquí se define el número de planos que se van a balancear, y por tanto el tipo de balanceo que se va a realizar. Para esto, la información de la figura 2 aclara gráficamente esa selección. De igual forma, este apartado recomienda la posición de los sensores.

Posteriormente se procede a la configuración de los sensores, a través de la información solicitada, tanto para los acelerómetros como para el tacómetro, evidenciado en la figura 3. Este proceso incluye la frecuencia de muestreo. 


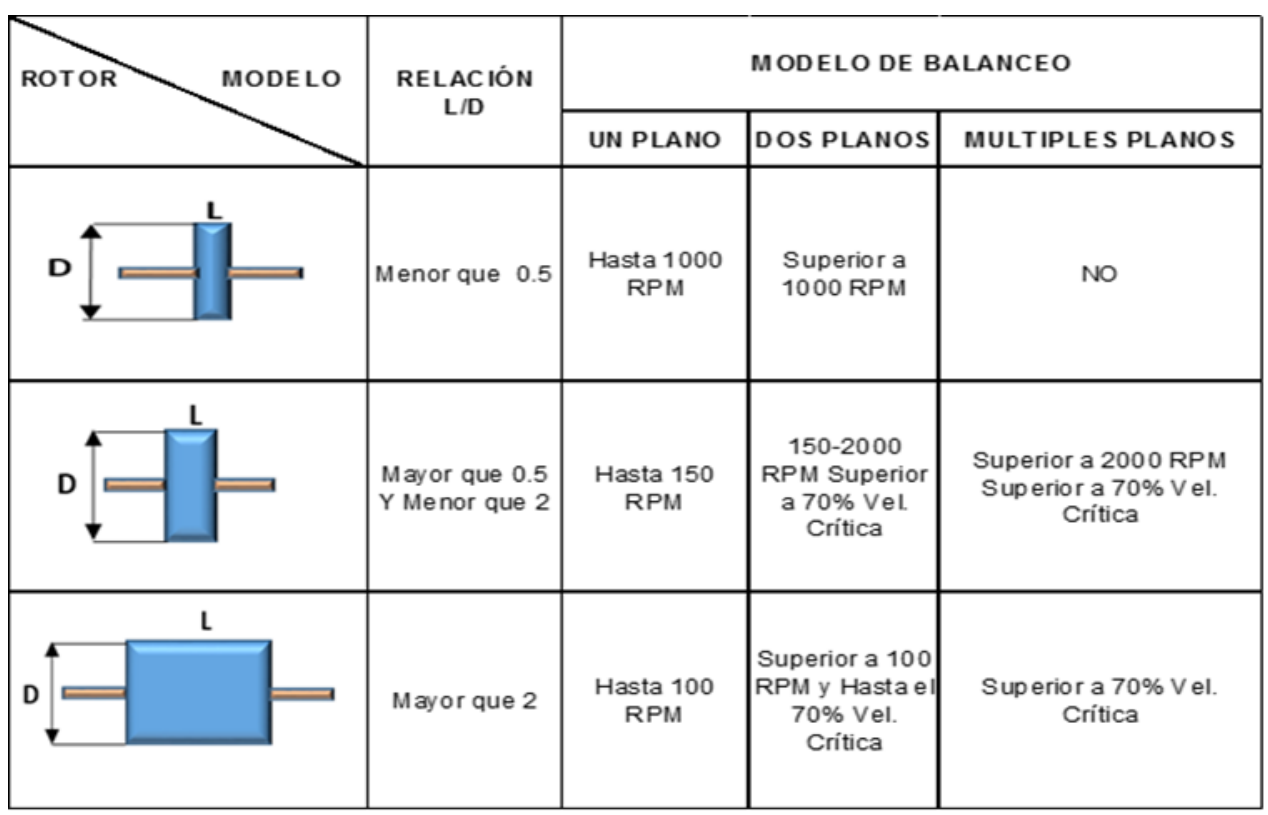

Figura 1. Modelos de balanceo.

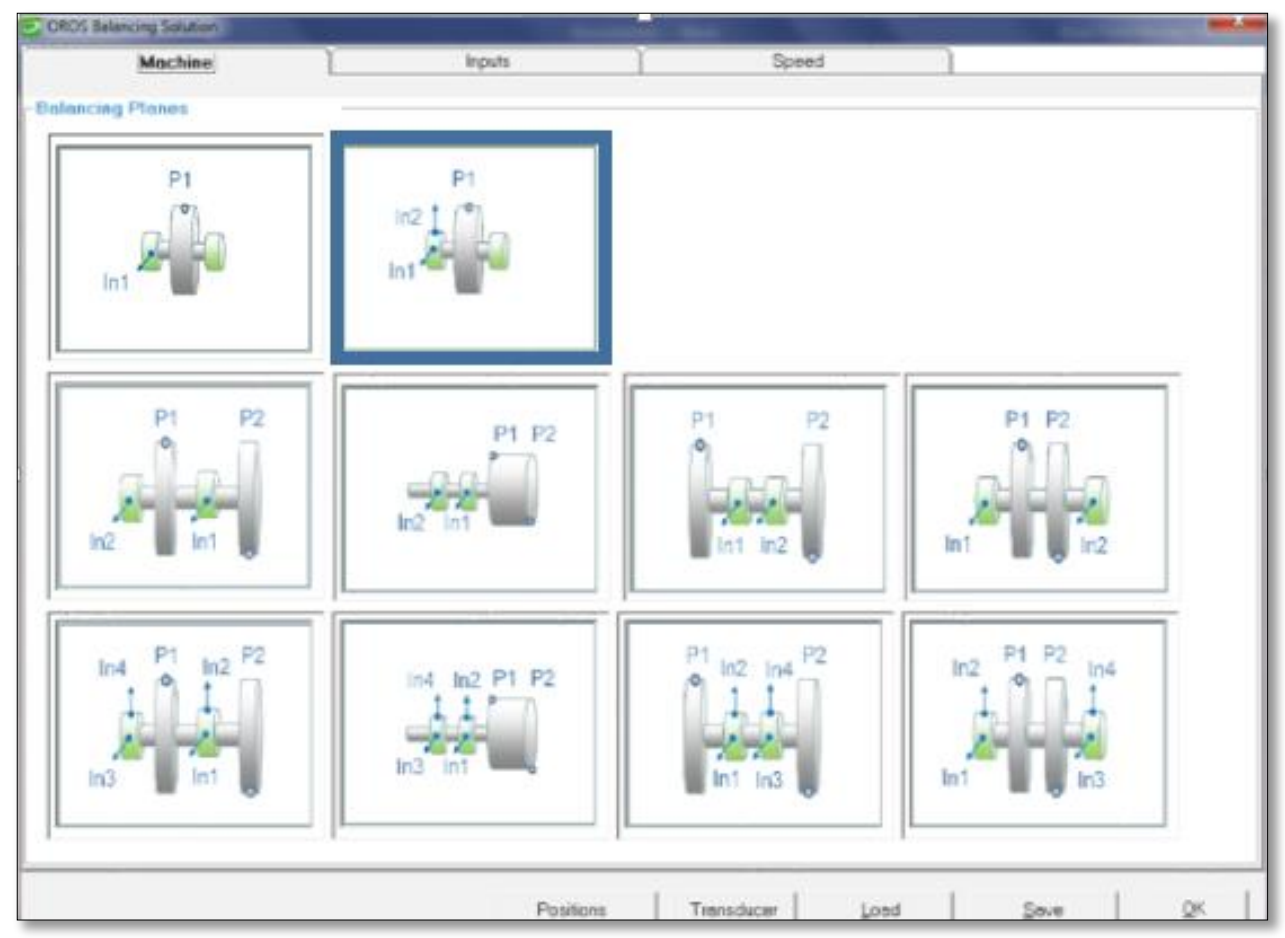

Figura 2. Configuración y ubicación de planos y acelerómetros que se van a trabajar. 


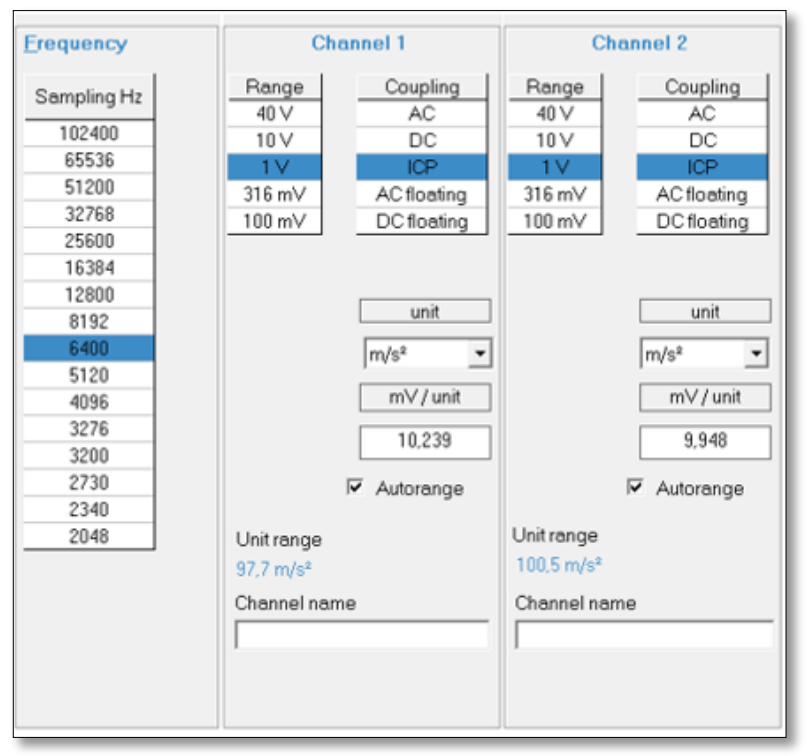

(a)

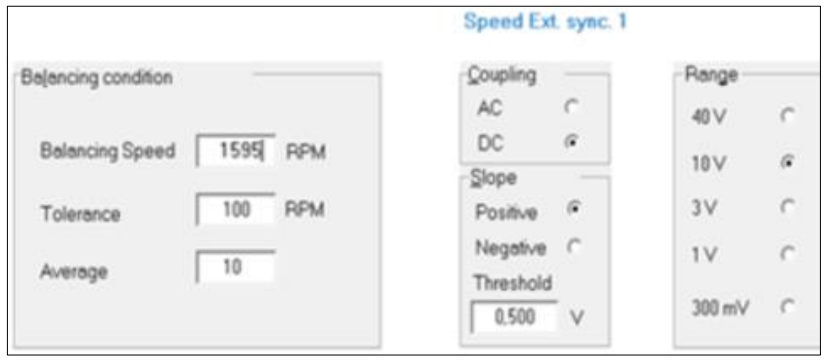

(b)

Figura 3. (a) Configuración de acelerómetros y frecuencia de muestreo, (b) configuración del tacómetro.

Configuración del rotor. Se ingresa la cantidad de planos, el número de las posiciones de las masas, el radio desde el centro de rotación a las posiciones de las masas, las unidades de acuerdo con el sistema internacional, o con el sistema inglés, la masa del rotor (esta masa debe ser dividida en el número de apoyos del rotor), la velocidad de rotación de los rotores, la calidad del balanceo y, por último, la metodología de trabajo, ya sea agregando o retirando masas de prueba (véase figura 4).

\subsection{Pasos del proceso}

El proceso está divido en tres grandes pasos, tal como se aprecia en la figura 5. En términos generales, en primero lugar se determina el estado inicial de balanceo del sistema, esto permite definir si se requiere realizar el balanceo; en segundo lugar, se introducen, de forma controlada, masas de desbalanceo, fundamentales para determinar tanto la masa de desbalanceo como su posición; en tercer lugar, se realizan los aportes o extracción de masa para balancear, según lo definido en el segundo paso, y por último lugar, se determina si se cumple con los niveles permitidos de desbalanceo, o si se hace necesario realizar nuevamente el proceso.

\subsection{Evaluación final del resultado del balanceo}

El objetivo de la prueba es disminuir el nivel de excentricidad de desbalanceo, señalado con el vector azul de la figura 6. El círculo concéntrico azul representa el desbalanceo residual admisible. Por tanto, el vector de excentricidad después de un proceso de balanceo satisfactorio debería quedar dentro de estos límites. La ventana mostrada en la figura 6 es una herramienta intuitiva que permite evaluar el resultado del proceso.

Finalmente, el proceso se valida con el análisis de vibraciones, en el que se debe evidenciar la disminución de la amplitud de vibración, en el espectro en frecuencias, del primer armónico (1X) principalmente, y el cumplimiento de la norma. 


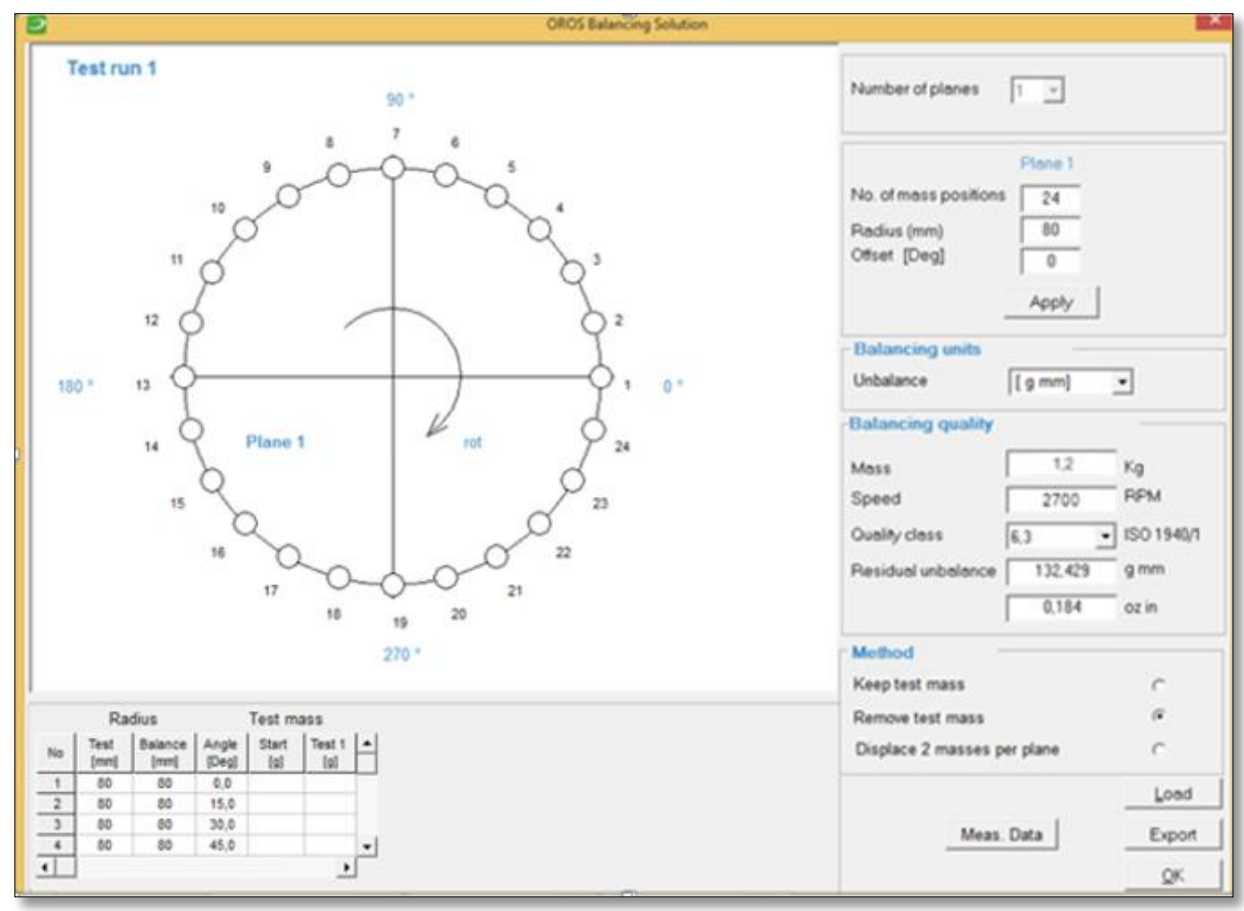

Figura 4. Configuración del rotor.

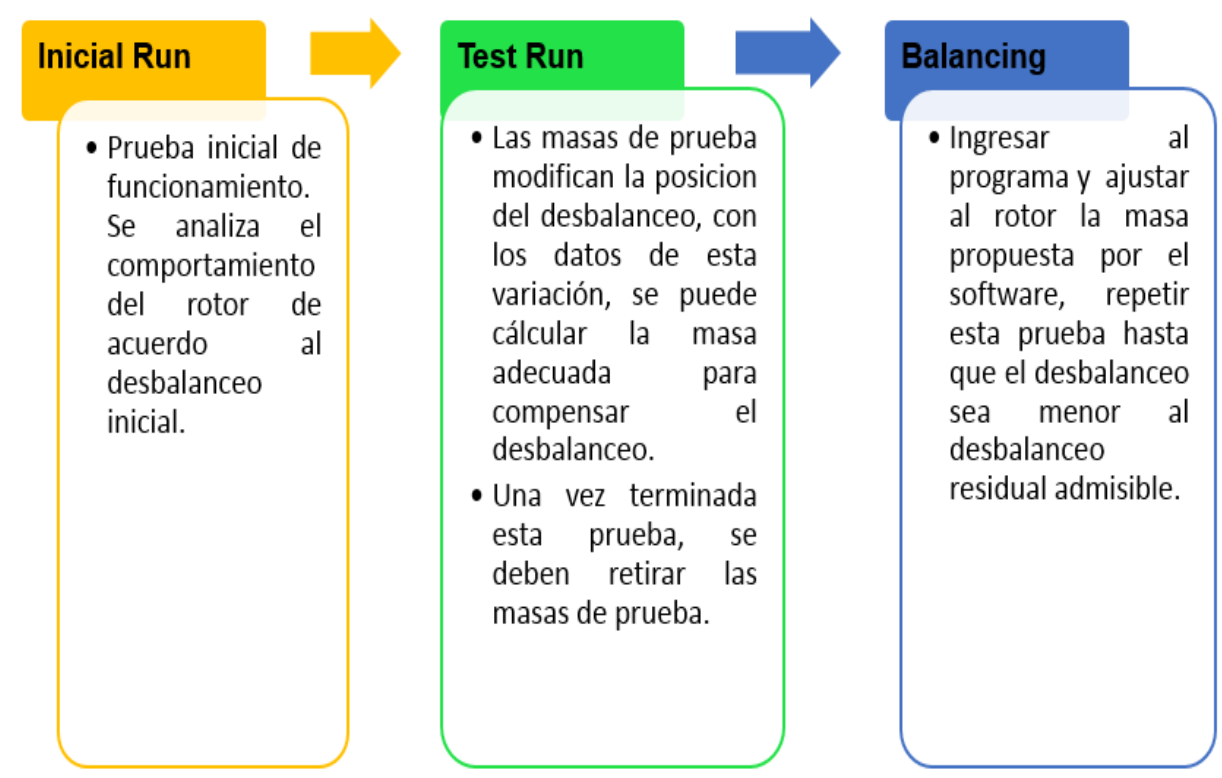

Figura 5. Metodología de trabajo en balanceo de rotores. 


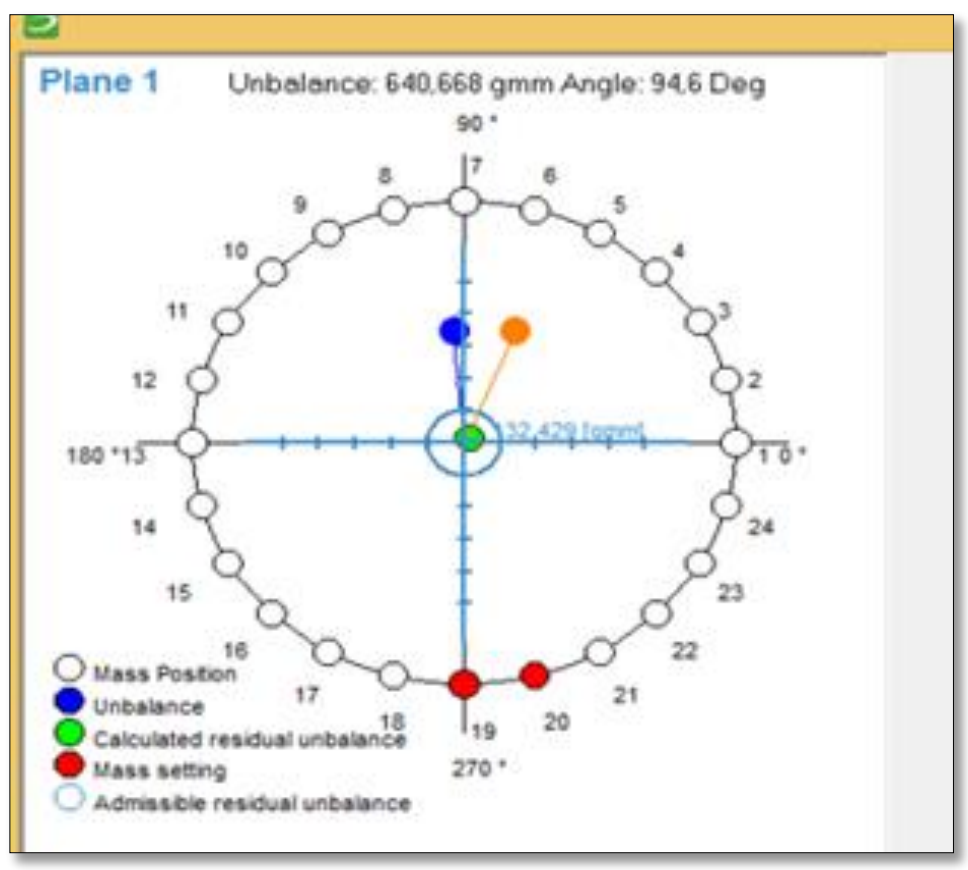

Figura 6. Representación gráfica de la ejecución del balanceo.

\section{Validación experimental de la metodología}

La validación de la metodología, empleando el sistema de adquisición OROS 35 y sus aplicaciones, se realiza para las diferentes opciones de balanceo descritas en el apartado 2. Los diferentes casos estudiados se han reproducido en diferentes bancos de ensayos de vibraciones mecánicas. De igual forma, se realizó la validación de la metodología y las herramientas para balanceo en maquinaria tipo industrial. En los análisis realizados se establece la condición de operación tanto para el sistema desbalanceado, es decir, antes de aplicar el proceso de balanceo, como para el instante posterior al proceso, con el objeto de evaluar su efectividad.

\subsection{Caso I. Balanceo estático de un rotor con un disco}

Para la reproducción de este caso su utilizó el banco de ensayos que se muestra en la figura 7. Puesto que el banco de ensayos esta acondicionado para el estudio y análisis vibratorios, su condición operacional es óptima. Por lo tanto se le induce, de forma controlada, masas de desbalanceo en el disco.

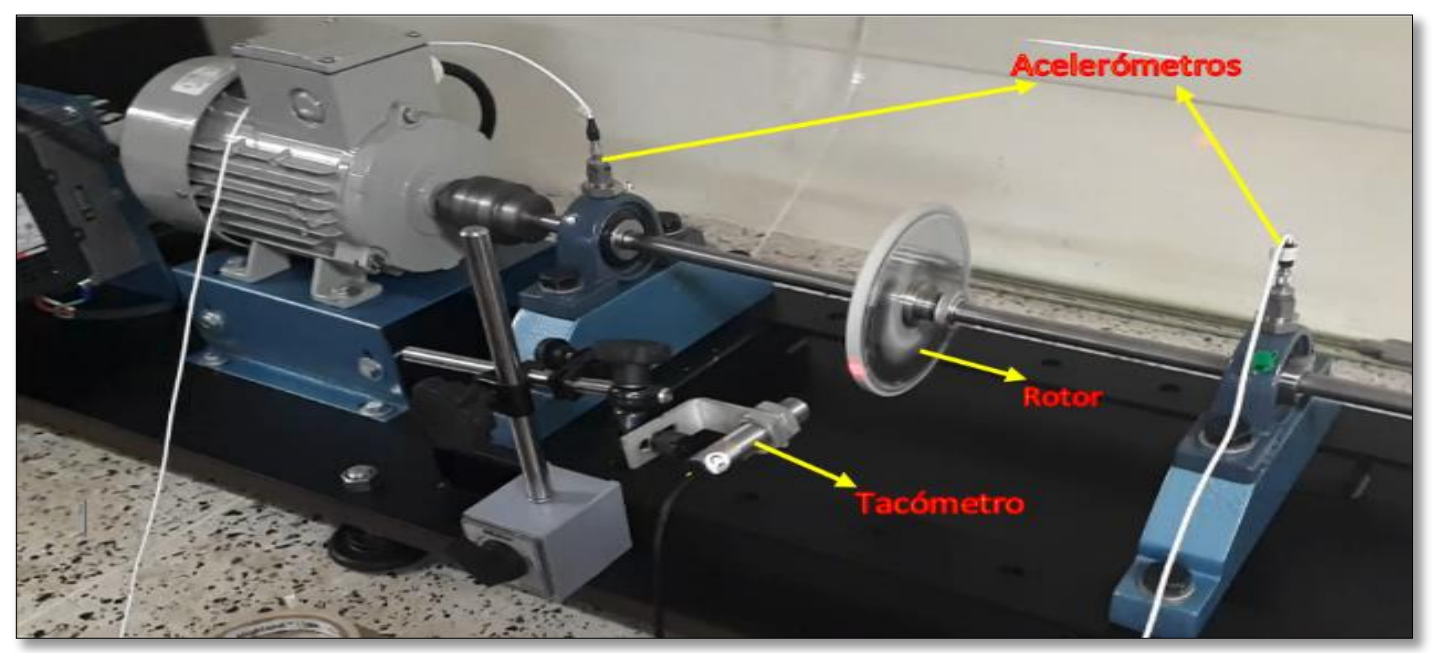

Figura 7. Banco de ensayos para el caso I. 


\subsubsection{Caso I "antes"}

Una vez agregada la masa aleatoria, se realiza el análisis de vibraciones para establecer la condición de operación del sistema. Como es evidente, se deben registrar amplitudes de vibración asociadas a problemas por desbalanceo.

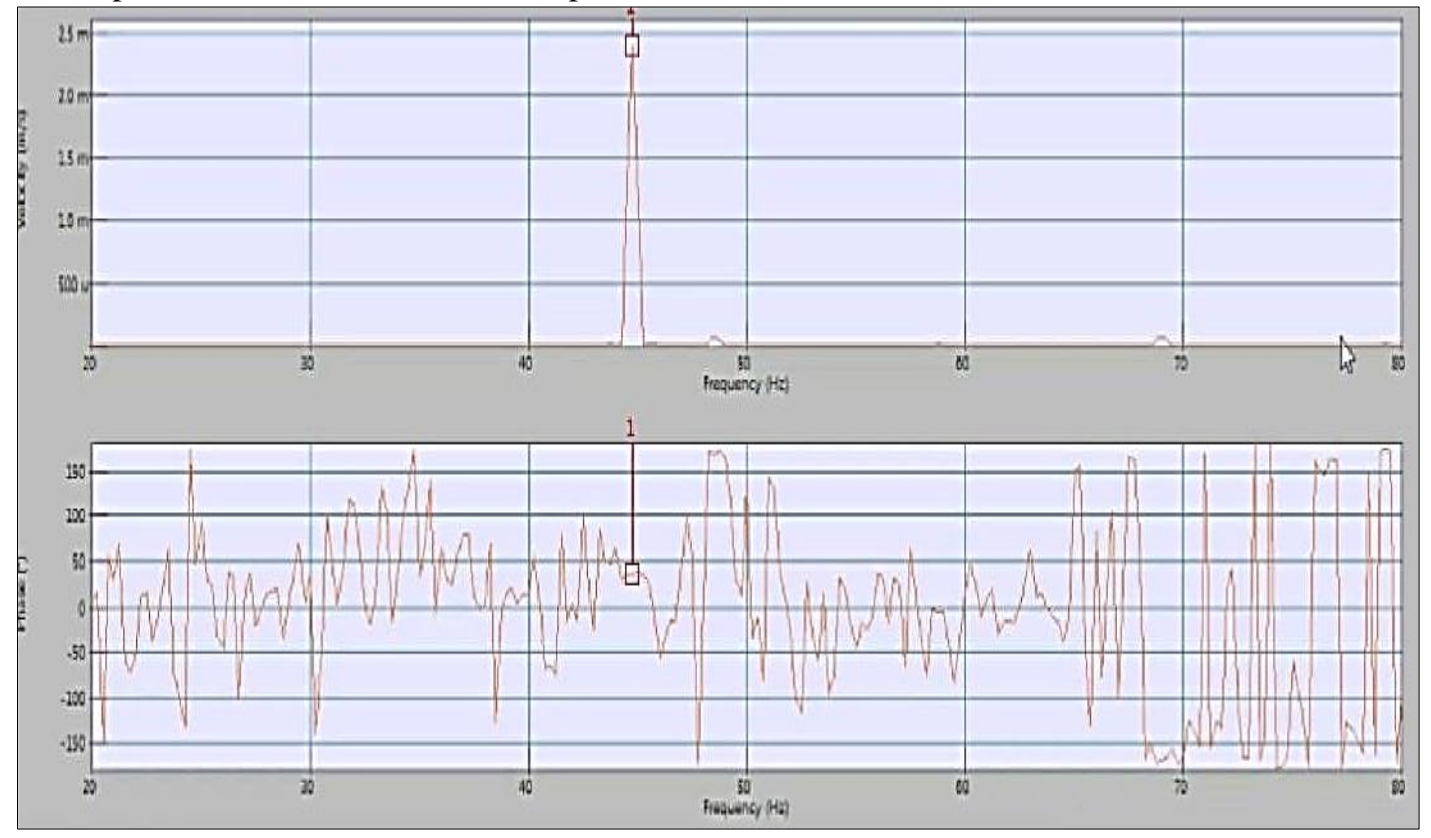

Figura 8. Espectro de frecuencia y ángulo de desfase de la señal obtenida en los puntos de apoyo del caso I, antes del balancearlo.

El espectro de frecuencia obtenido se muestra en la figura 8 , donde se evidencia un pico a una amplitud de 2.5 $[\mathrm{mm} / \mathrm{s}]$ y una frecuencia de $45[\mathrm{~Hz}]$, correspondiente al armónico primario de vibración (1x), dada la frecuencia de giro del rotor. Además de ello, el análisis de fase de este espectro, mediante la comparación de las señales de los dos acelerómetros ubicados en los dos soportes del eje, es aproximadamente igual a un desfase de $40^{\circ}$, lo que evidencia un desbalanceo estático en el rotor.

\subsubsection{Caso I "después"}

$\mathrm{Al}$ realizar el balanceo del banco de ensayos del caso I, a partir de las señales obtenidas en los acelerómetros y el desarrollo de la metodología previamente explicada, se obtuvo el espectro que se muestra en la figura 9. Como se puede observar, la disminución del pico a $45 \mathrm{~Hz}$ es notable, ya que descendió hasta un valor de $1.8[\mathrm{~mm} / \mathrm{s}]$, y dada la norma ISO 10816, que evalúa la vibración de una máquina mediante medidas en partes no rotativas, el banco de ensayos está dentro de las condiciones deseables de trabajo.

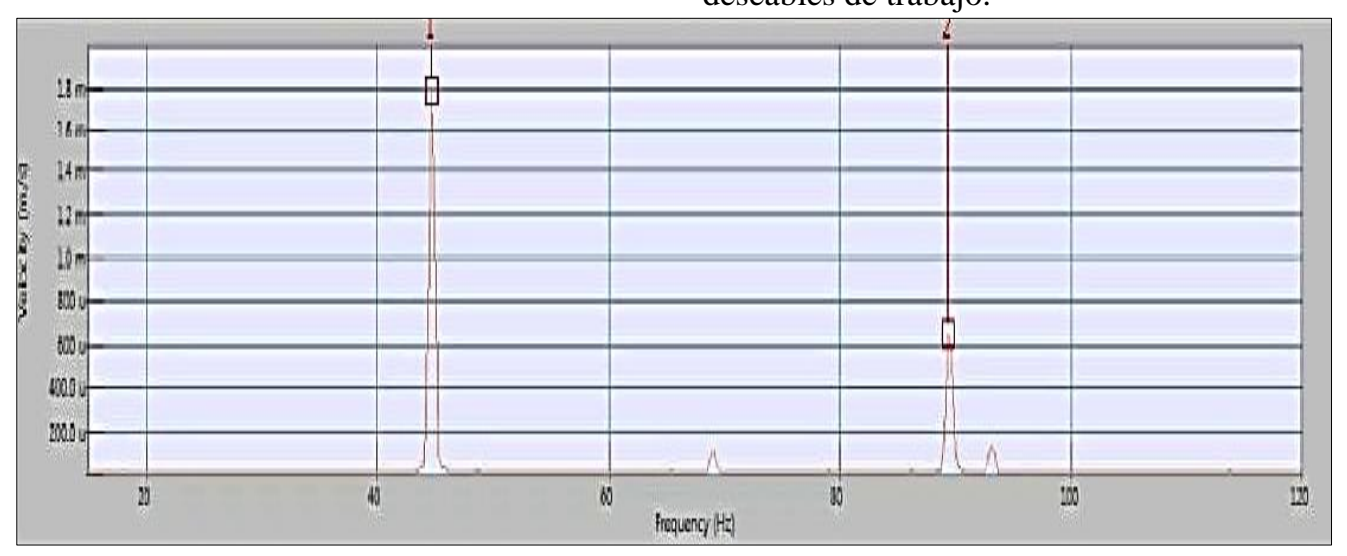

Figura 9. Espectro de frecuencia del caso I, después del balanceo. 


\subsection{Caso II. Balanceo cuasiestático de un rotor con dos discos}

Para este caso se emplea un banco de ensayos con dos discos y diferente topología del eje. El banco empleado se puede ver en la figura 10 .

\subsubsection{Caso II "antes"}

Al igual que en el caso I, se le indujo un desbalanceo agregando masas a los dos discos. El espectro de frecuencia que se obtuvo se muestra en la figura 11.

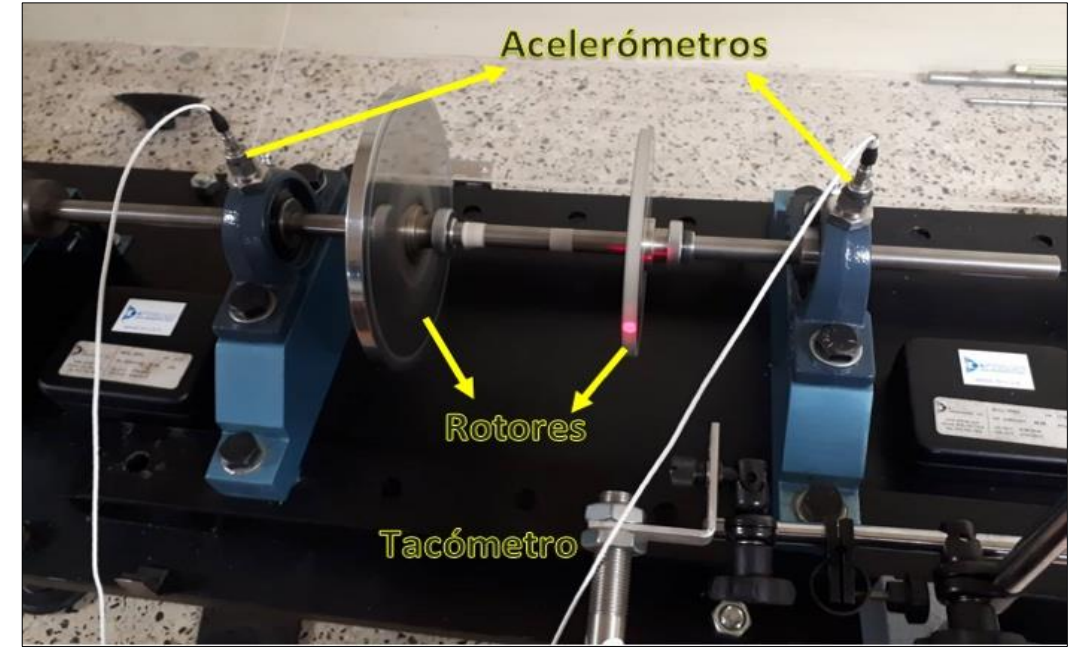

Figura 10. Banco de ensayos caso II.

Para este caso se varió la rotación del motor a $30 \mathrm{~Hz}$. Esta frecuencia es el valor del armónico primario. Este espectro evidencia un desbalanceo cuasiestático, dada la amplitud del pico a $30 \mathrm{~Hz}$, con un valor de $4.3[\mathrm{~mm} / \mathrm{s}]$ y un desfase entre las señales de los acelerómetros de aproximadamente $50^{\circ}$.

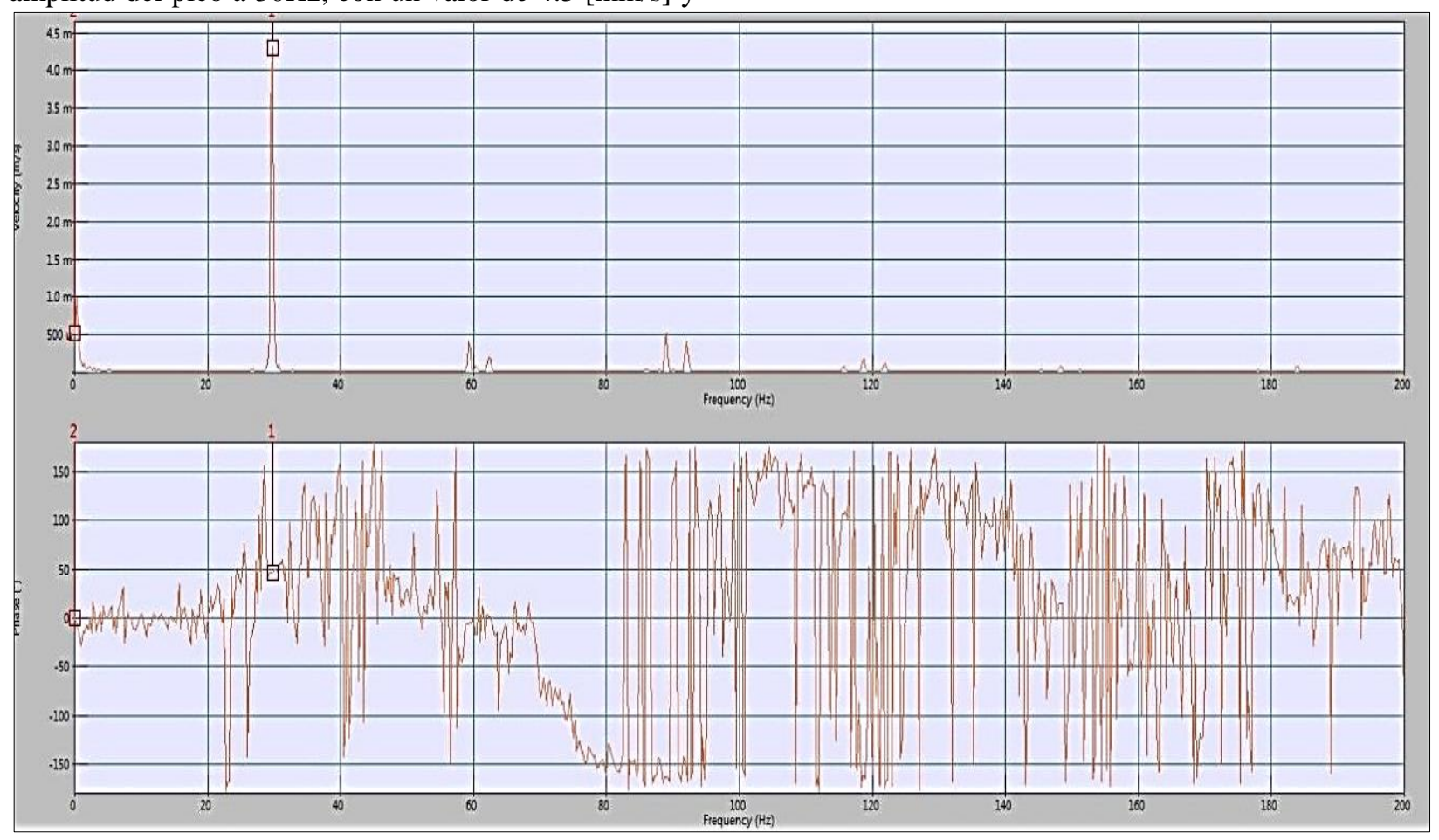

Figura 11. Espectro de frecuencia y ángulo de desfase de la señal obtenida en los puntos de apoyo del caso II, antes del balancearlo. 


\subsubsection{Caso II "después}

Una vez realizada la metodología de balanceo se obtuvo el resultado de cada uno de los dos planos. Para el primer plano se obtiene un resultado de desbalanceo de $59.900[\mathrm{~g} * \mathrm{~mm}]$, que se encuentra por debajo del desbalanceo residual admisible, el cual es de 83.556[g*mm], según la norma ISO 1940. Para el segundo plano se logra reducir el desbalanceo de un valor de $1153.893[\mathrm{~g} * \mathrm{~mm}]$ a un valor de $110.799[\mathrm{~g} * \mathrm{~mm}]$, muy cercano al desbalanceo residual admisible.

El espectro obtenido una vez realizado el balanceo se muestra en la figura 12. Allí se evidencia la reducción del pico a $30 \mathrm{~Hz}$, de $4.3[\mathrm{~mm} / \mathrm{s}]$ a $0.9[\mathrm{~mm} / \mathrm{s}]$. El resultado es bueno en una evaluación de estado funcional del banco de ensayos.

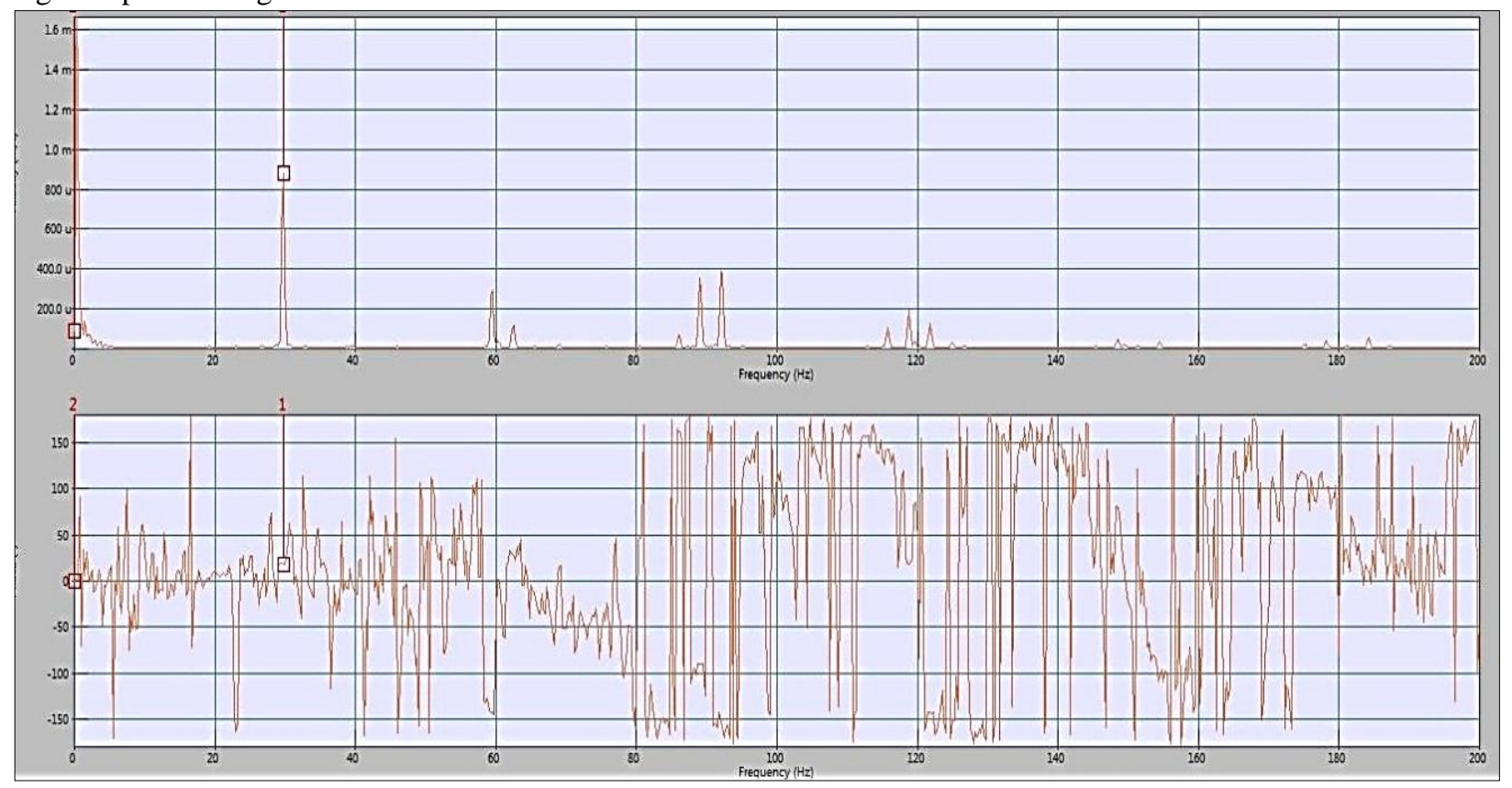

Figura 12. Espectro de frecuencia y ángulo de desfase de la señal obtenida en los puntos de apoyo del caso II, después de balancearlo.

\subsection{Caso III. Balanceo dinámico de un rotor con 3 discos}

Para la reproducción de este caso se utilizó un banco de ensayos que se muestra en la figura 13.El banco consta de un eje con 3 discos equidistantes acoplados, y dos soportes. La configuración del software para este caso difiere de los anteriores, puesto que el objetivo, al balancear el rotor con tres discos, es generar un balanceo en múltiples planos.

\section{Comportamiento dinámico en rotores flexibles y su relación en el balanceo}

Es importante tener en cuenta que los rotores flexibles tienden a flexionarse bajo la acción de las fuerzas dinámicas producidas por el desbalance. Tal es el caso de los rotores de turbinas de vapor y de gas. Esto hace imprescindible balancear los rotores flexibles en múltiples planos, para lo cual es necesario que durante el balanceo el rotor sea operado en condiciones similares a las del servicio, es decir, como mínimo por encima de su primera velocidad crítica, debido a que, si los pesos de balanceo no se colocan exactamente en las posiciones de balanceo, entonces se generarán pares de fuerzas dinámicas que desbalancearán el rotor, una vez alcance su velocidad de operación.

Para el presente caso existe una relación entre la velocidad crítica y el número de planos requeridos para balancear.

\subsubsection{Procedimiento previo al balanceo del caso III}

El proceso descrito en el apartado 3.2 es aplicado en plenitud para el balanceo. Este proceso requiere varias corridas para lograr un óptimo resultado. Al igual que en los casos anteriores, se registra la señal de vibraciones antes de aplicar la metodología, para compararla con la medida de vibraciones posterior al balanceo. Es así como se verifica la efectividad del proceso. El fallo se induce mediante masas aleatorias roscadas en cada uno de los discos del banco mostrado en la figura 13. 
En la figura 14, se observa una amplitud de 37 [mm/s] del pico $1 \mathrm{X}$ a una frecuencia de $40 \mathrm{~Hz}$. Se evidencia un alto grado de desbalanceo dadas las masas que se agregaron. Es importante resaltar que, en este caso, el banco presenta desgaste de los rodamientos; por lo tanto las señales de vibraciones están afectadas por este otro fallo, lo cual supone una dificultad para tener en cuenta durante la aplicación de la metodología.

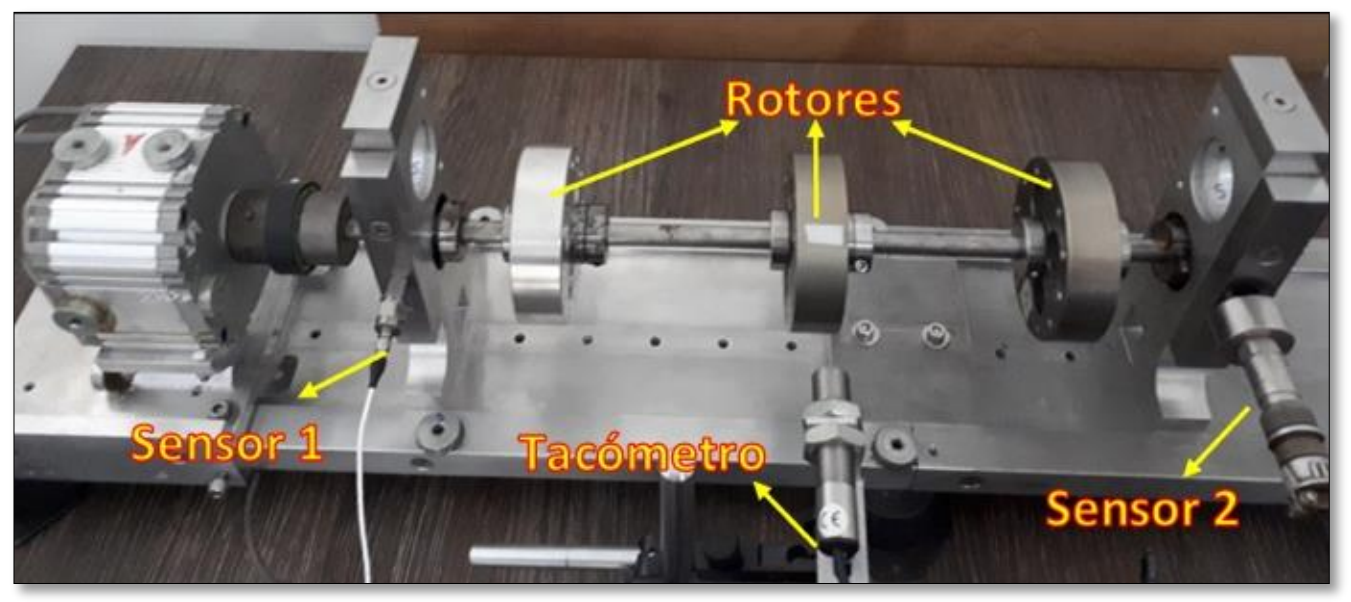

Figura 13. Banco de ensayos del caso III.

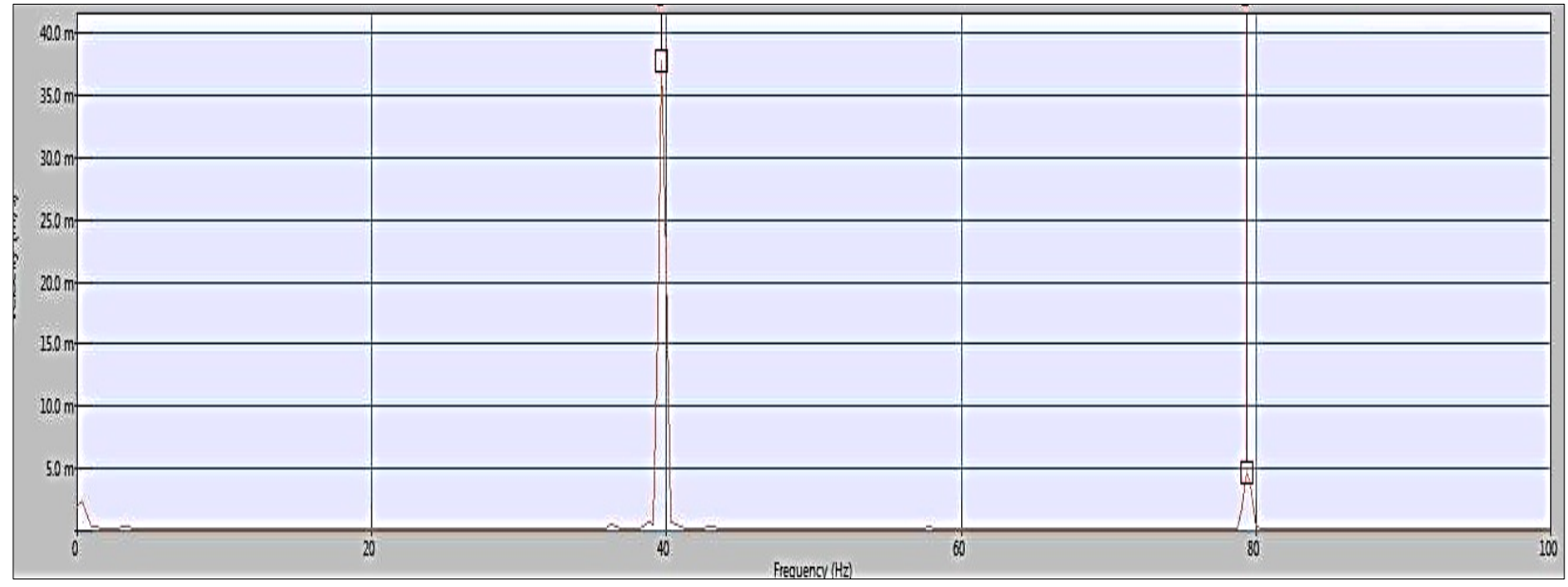

Figura 14. Espectro de frecuencia del caso III, antes de ejecutar el balanceo dinámico.

\subsubsection{Resultados del balanceo para el caso III}

Una vez realizados los procedimientos correspondientes al balanceo en múltiples planos, se obtiene la figura 15, que evidencia la reducción del desbalanceo. La figura 16 permite evidenciar la corrección del desbalanceo mediante una gráfica en coordenadas polares.

Finalmente, en la figura 17 se observa el espectro en frecuencia después del balanceo. En esta se puede apreciar que la amplitud del primer armónico se redujo de forma importante. No obstante, bajo la norma ISO 10816, la máquina no está en condiciones de operar, porque su amplitud excede los niveles permitidos. Esto está asociado a la condición de desgaste de los rodamientos. A pesar de ello, se avala la metodología, ya que el efecto del balanceo multiplano sobre la amplitud del primer armónico fue importante.

\subsection{Caso IV. Balanceo a una máquina de tipo industrial|}

Para este caso se realizó el balanceo a una máquina industrial, específicamente al rotor de un ventilador centrífugo que se encuentra acoplado a un motor eléctrico mediante un sistema de transmisión de potencia por banda (véase figura 18). 


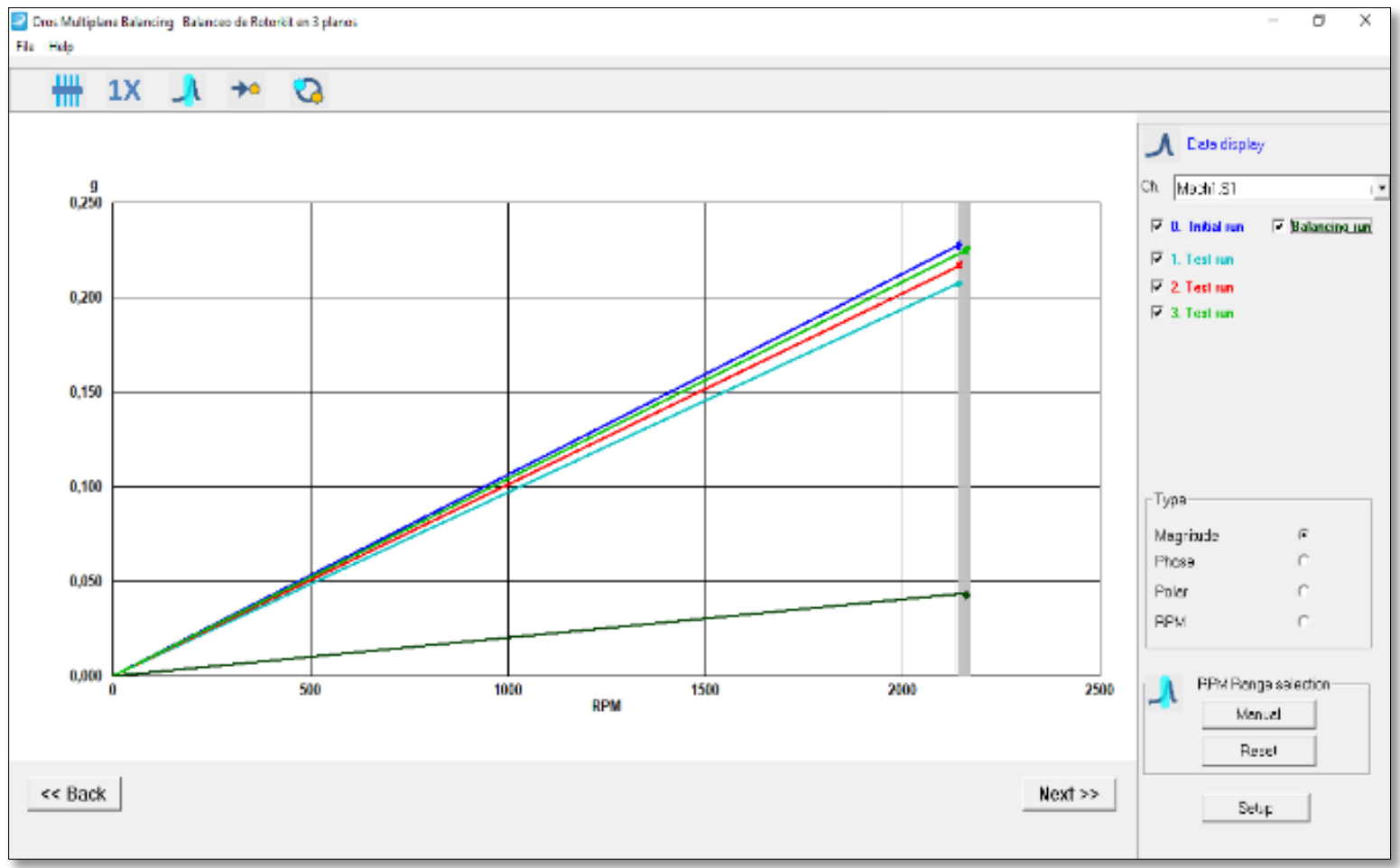

Figura 15. Gráfica lineal que representa la reducción del desbalance en el caso III.

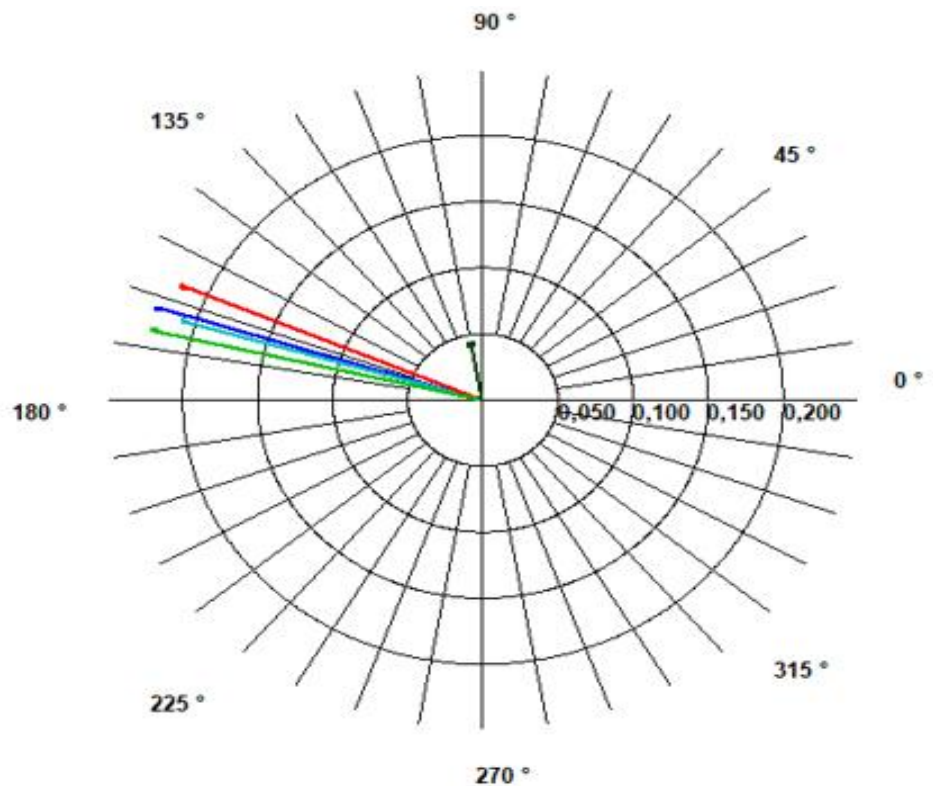

Figura 16. Gráfica polar que representa la reducción del desbalance en el caso III. 


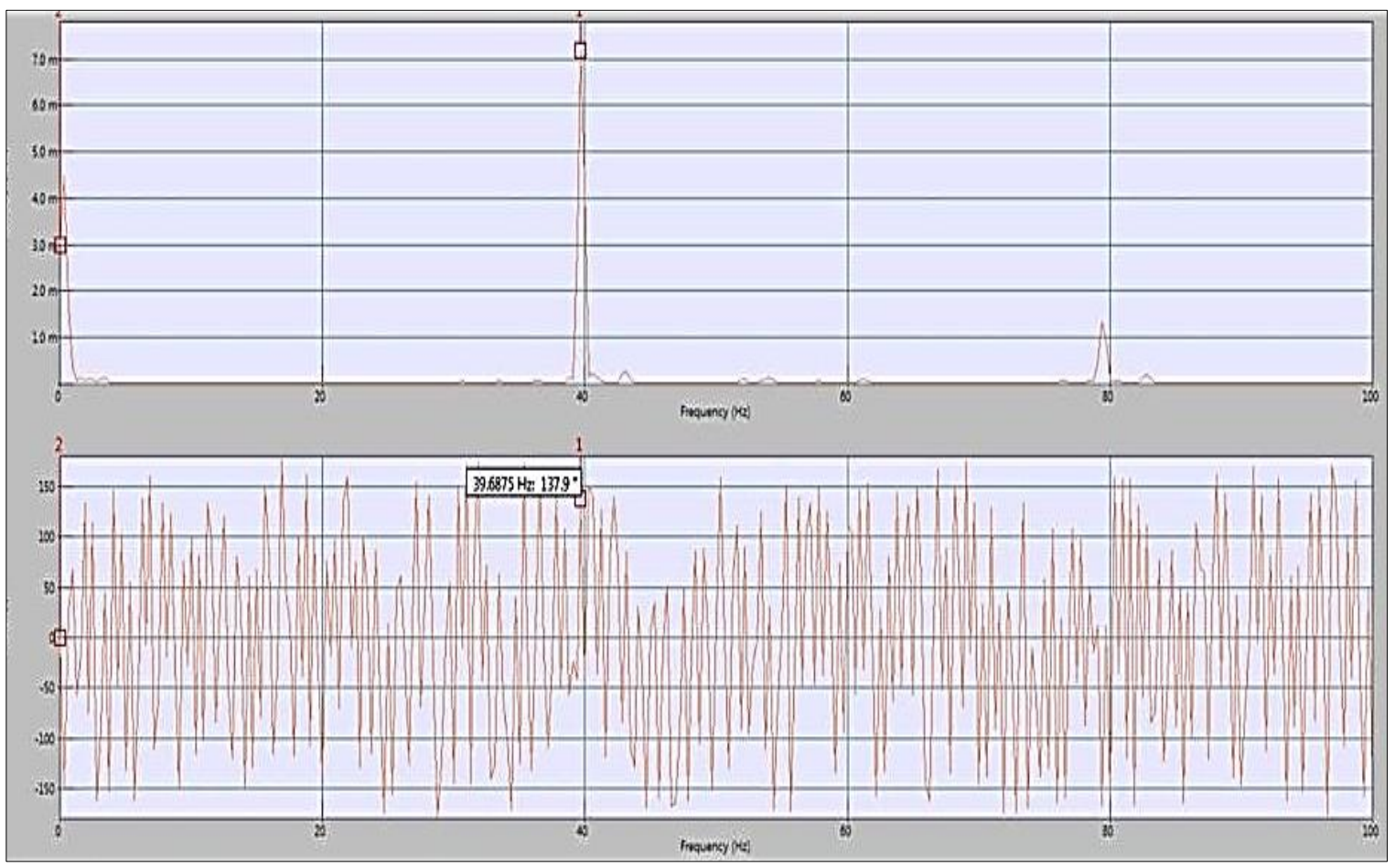

Figura 17. Espectro de frecuencia y ángulo de desfase de la señal obtenida en los puntos de apoyo del caso III, después de balancearlo.

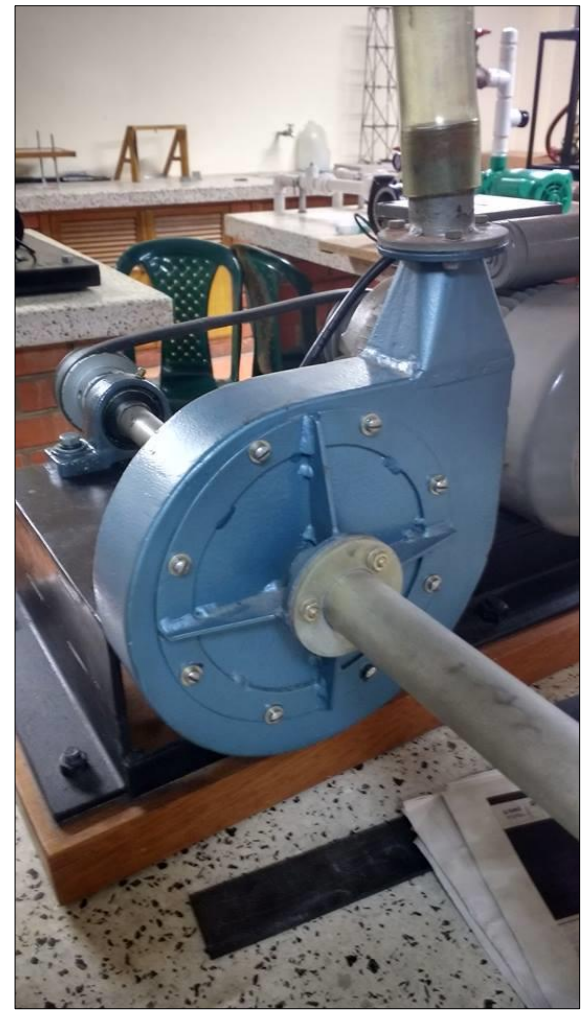

Figura 18. Banco máquina de tipo industrial para el caso IV.

\subsubsection{Características operacionales y de montaje del caso IV}

El ventilador gira a una velocidad estable de 4360 [RPM], medida en la polea conducida. La figura 19 muestra la ubicación de los acelerómetros en posición axial y radial, puesto que, a diferencia de los casos anteriores, la medición axial es necesaria en este tipo de montaje, debido a que el rotor se encuentra en voladizo.

\subsubsection{Procedimiento de balanceo para máquinas industriales del tipo del caso IV}

En algunos casos, como la construcción de turbinas hidráulicas, de vapor o de gas, las aspas del ventilador se fabrican por separado y luego se sueldan, o insertan en ranuras en posiciones regularmente espaciadas alrededor de la periferia de un cilindro. Debido a las imperfecciones de fabricación, las cuchillas no son idénticas, y sus pesos, así como las ubicaciones de sus centros de gravedad, pueden variar.

Un paso previo en equipos industriales es marcar la locación de los puntos de prueba con una secuencia de puntos a intervalos regulares alrededor del perímetro del rotor (véase figura 20). 


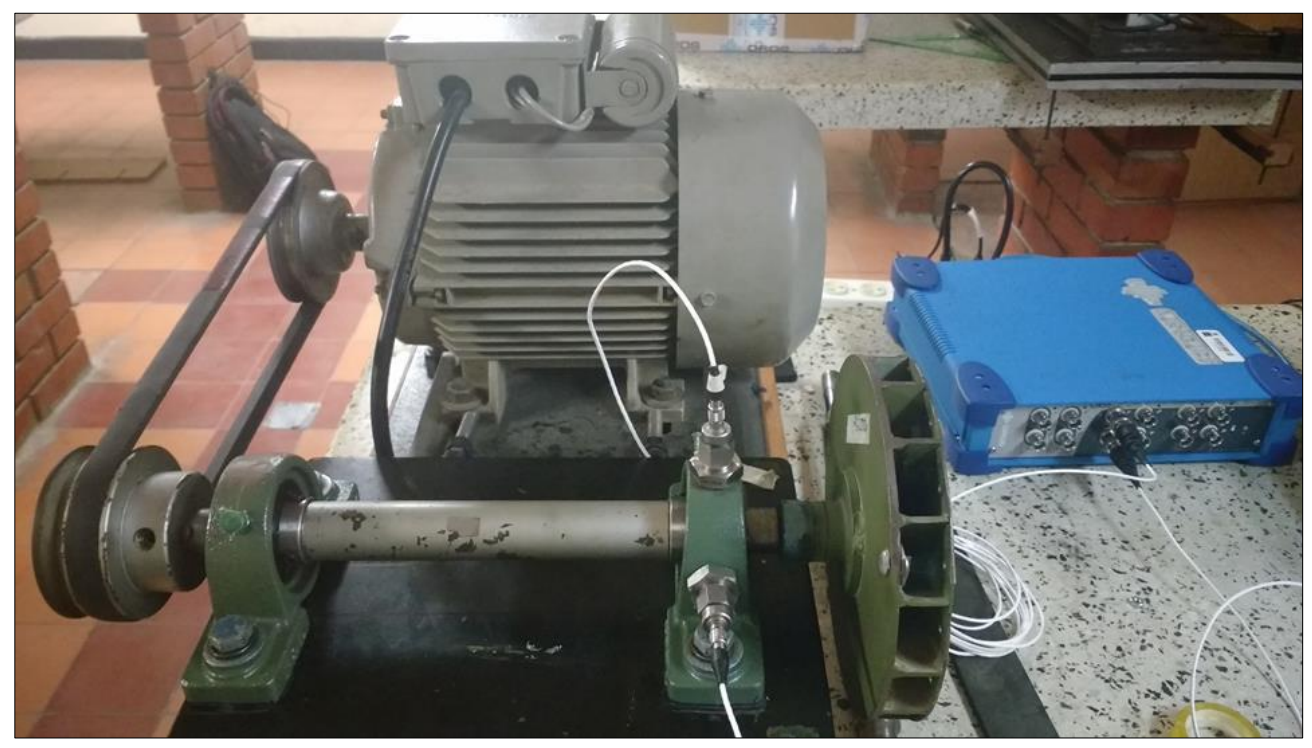

Figura 19. Montaje del de acelerómetros para el caso IV.

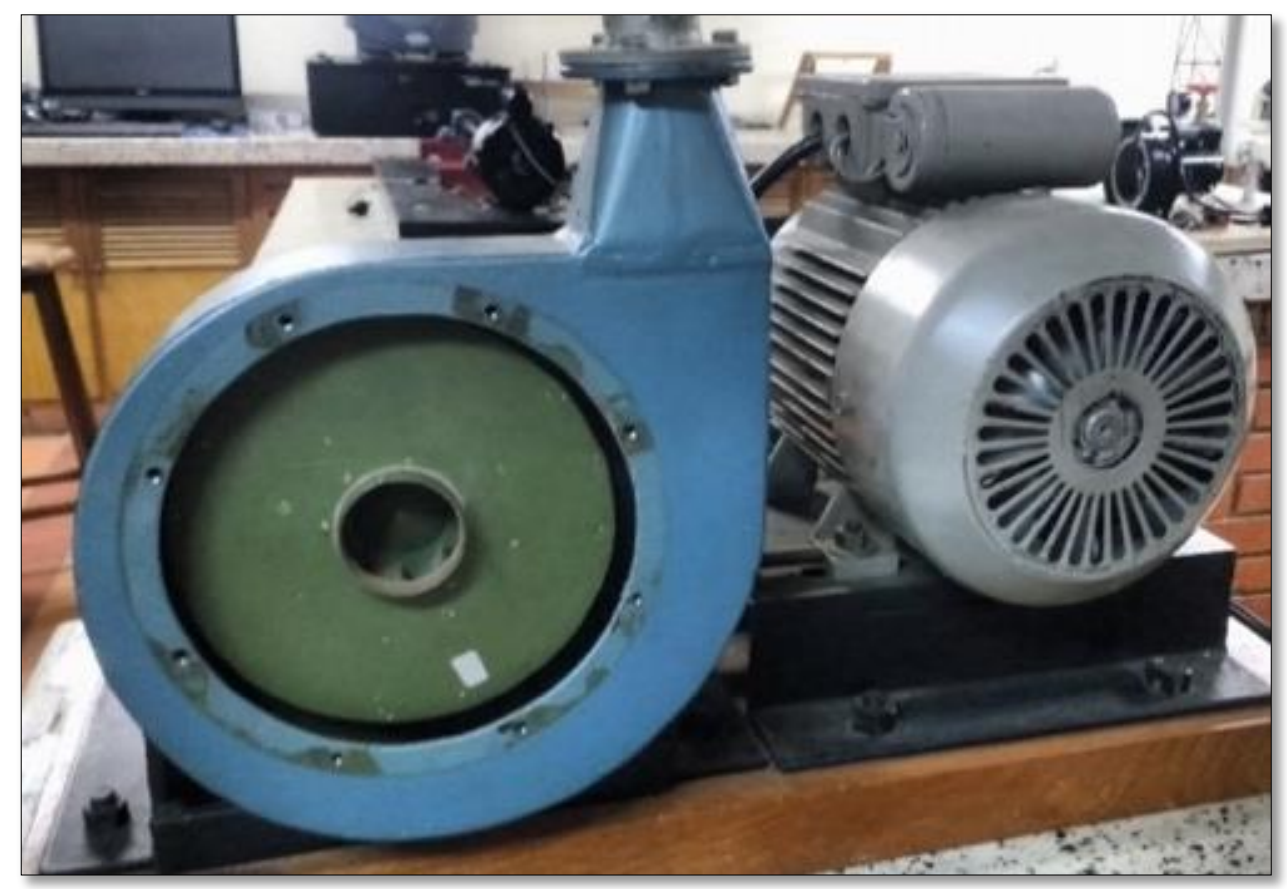

Figura 20. Ubicación de marcas en el rotor del ventilador centrifugo para la adición de masas de balanceo.

\subsubsection{Caso IV "antes"}

La figura 21 reporta el espectro en frecuencia antes de realizar los respectivos ajustes de balanceo. En ella, se identifica un pico de vibración asociado al desbalanceo, con un amplitud de $11.73[\mathrm{~mm} / \mathrm{s}]$ a la frecuencia de rotación, $72.5[\mathrm{~Hz}]$.

\subsubsection{Balanceo del rotor}

Se procede a aplicar la metodología de balanceo para un rotor en voladizo, configuración asociada al presente caso. En la figura 22 se puede apreciar el valor actual de excentricidad, y los valores de masas y sus posiciones para corregir ese fallo. 


\section{RUII, REVISTA UIS \\ INGENIERÍAS}

Otra información que proporciona la figura 22 es el pronóstico del balanceo (punto verde dentro del circulo azul) con las masas de 1 [g] y el desbalanceo residual de 195,9 [g*mm.]. El valor de desbalanceo residual permisible para este caso es de 13,798 [g*mm].

\subsubsection{Resultados del balanceo del caso IV}

Después de realizar la primera prueba de balanceo se observa que el desbalanceo se redujo a 57.236 [g*mm]. No se logra ubicar el desbalanceo por debajo del desbalanceo residual admisible, como se aprecia en la figura 23. Se realiza nuevamente el proceso, se obtienen nuevas masas en nuevas ubicaciones hasta concluir el proceso.

Se toma los espectros de frecuencia y, como se observa en la figura 24, se obtuvo una reducción en el pico $1 X$ hasta un valor de $3.72[\mathrm{~mm} / \mathrm{s}]$ a $72.5[\mathrm{~Hz}]$. La reducción evaluada con la norma arroja un resultado de condición aceptable de operación de la máquina.

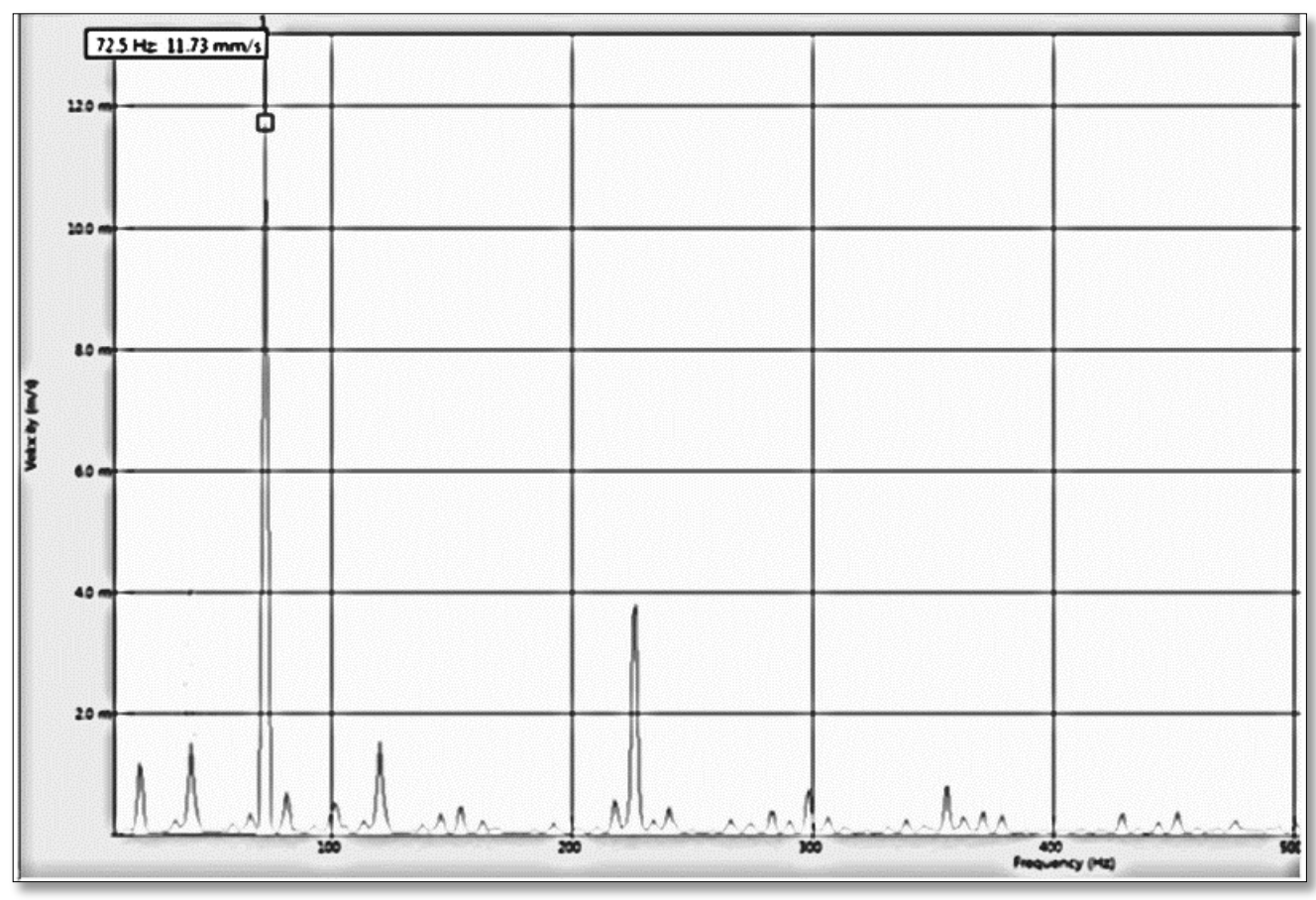

Figura 21. Espectro de frecuencia del caso IV, antes del balanceo. 


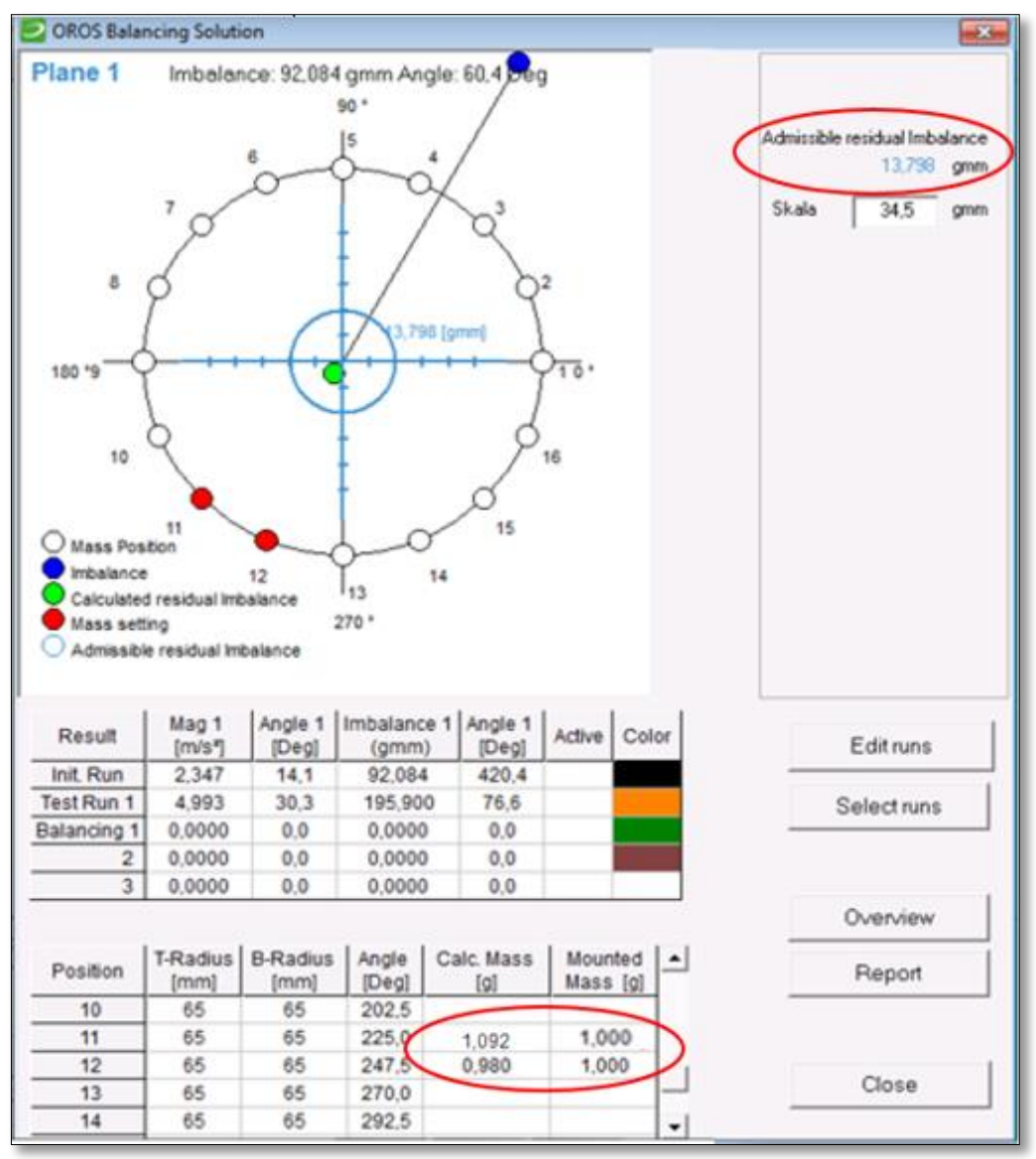

Figura 22. Ejecución del balanceo del rotor del ventilador centrífugo. 


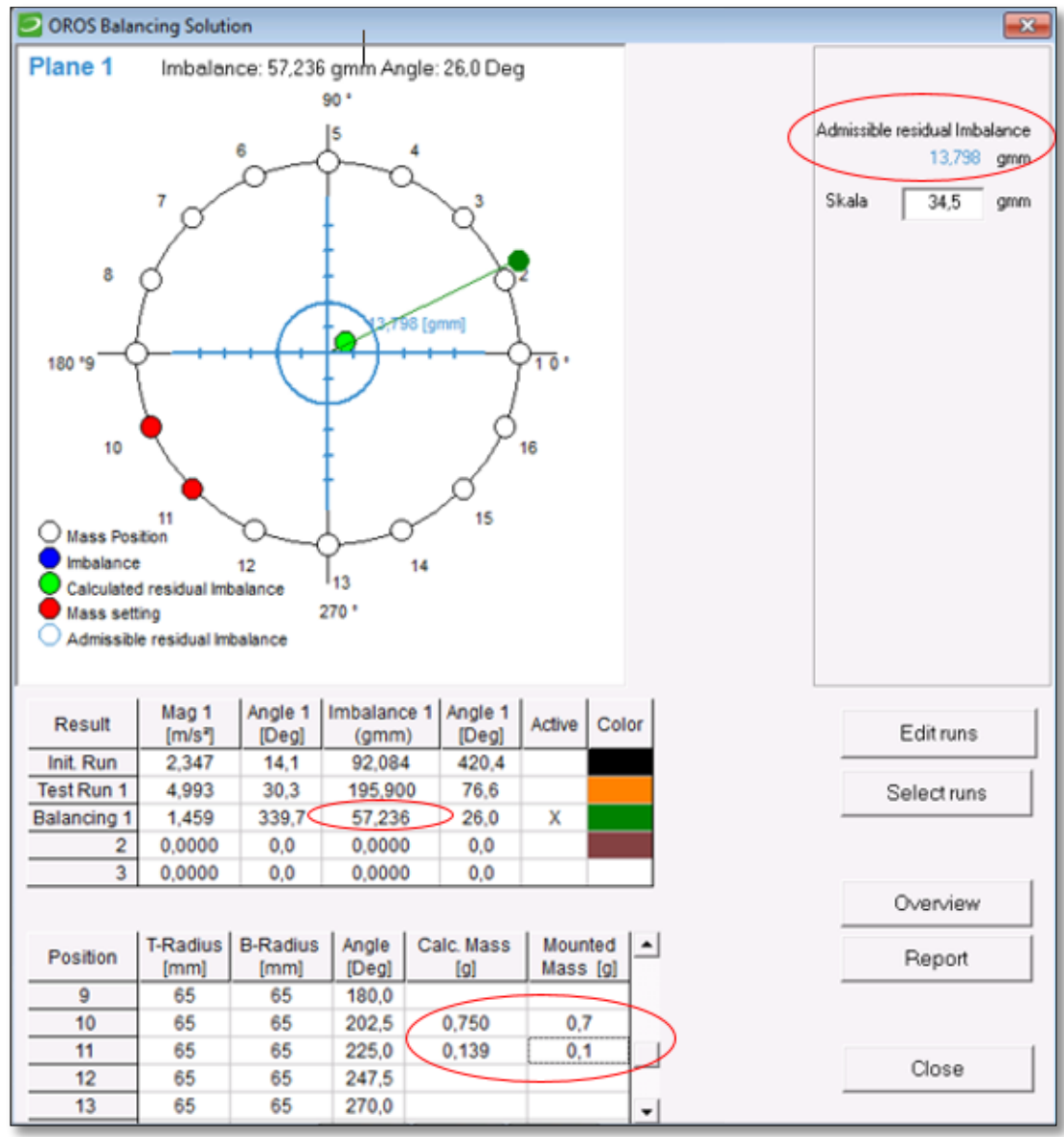

Figura 23. Resultados del balanceo residual para el caso IV.

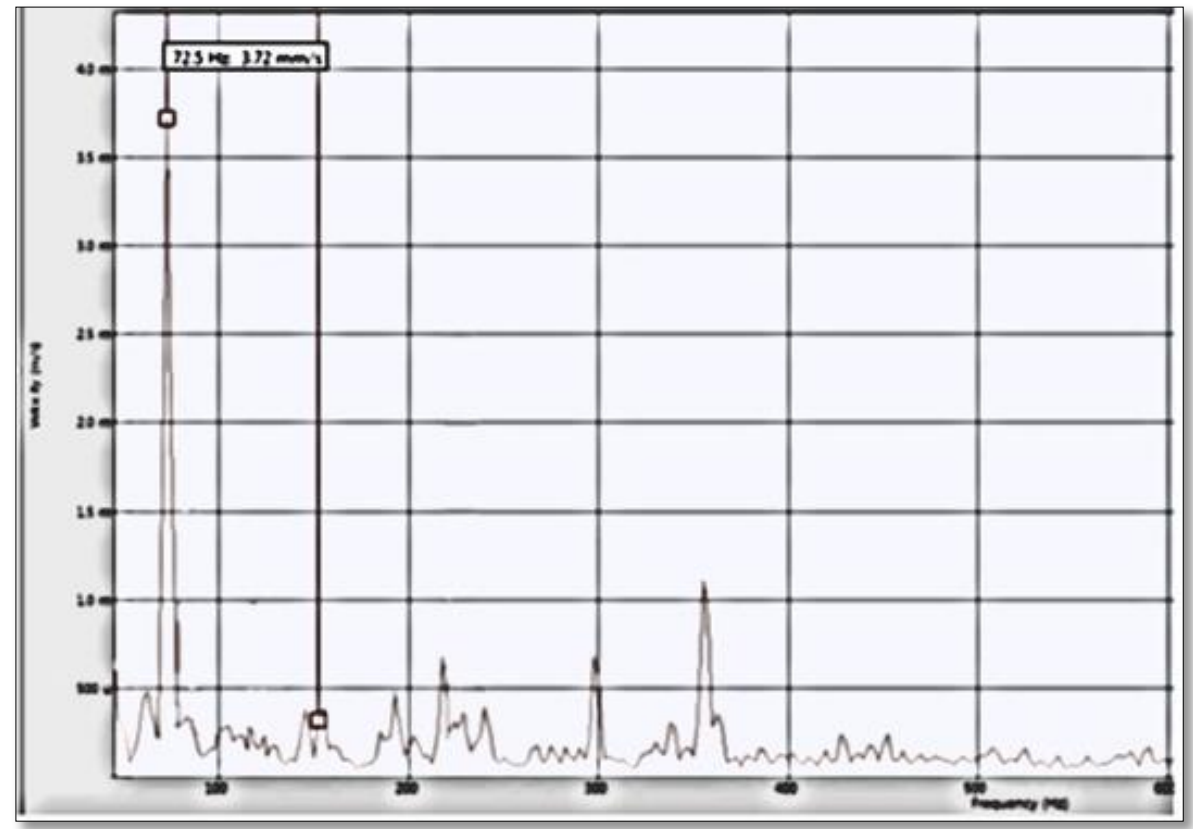

Figura 24. Espectro de frecuencia del caso IV, después generar el balanceo. 


\section{Conclusiones}

Se implentó una metodología para el balanceo estático y dinámico de rotores, a través de un sistema de adquisición de vibraciones (OROS 35) y una aplicación informática que permite la estimación y la ubicación de la masa de balanceo.

La metodología ha sido validada de forma experimental, empleando cuatro casos diferentes de estudio que comprenden el balanceo estático, el balanceo dinámico en dos planos y el balanceo dinámico en múltiples planos.

La validación de la metodología se ejecutó de forma experimenal tanto en bancos de laboratorio para el estudio de vibraciones mecánicas como en una máquina de tipo industrial.

Los resultados del proceso han sido satisfactorios en todos los escenarios verificados. Estos resultados fueron contrastados con respecto a la norma.

\section{Bibliografía}

[1] E. Estupiñán P., C. San Martín, and L. Canales M., "Desarrollo de un instrumento virtual para el balanceamiento dinámico de rotores," Ingeniare Rev. Chil. Ing., vol. 14, 2, pp. 146-152, 2006.

[2] H. G. Sánchez A., "Modelo analítico para el estudio de la torsión en rotores flexibles," Rev. UIS Ing,. vol. 9, no. 1,2010 .

[3] H. G. Sánchez Acevedo, J. Uscátegui, and S. Gómez, "Metodología para la detección de fallas en una estructura entramada metálica empleando las técnicas de análisis modal y PSO," Rev. UIS Ing., vol. 16, no. 2, pp.43-50, 2017.

DOI:

https://doi.org/10.18273/revuin.v16n2-2017004

[4] J. M. Pachón, O. A. González, and H. G. Sánchez, "Detección de daños en una armadura unidimensional por medio del algoritmo de optimización de la luciérnaga y elementos finitos," Av. Investig. en Ing., vol. 13, no. 1, pp. 1-7, 2017.

[5] H. G. Sanchez, D. M. C. Marulanda, and E. G. Florez, "Application of Vibration Based Damage Identification Techniques on Metallic Structures," Adv. Mater. Res., vol. 875-877, pp. 875-879, 2014.

[6] M. Hutterer, G. Kalteis, and M. Schrödl, "Redundant unbalance compensation of an active magnetic bearing system," Mech. Syst. Signal Process., vol. 94, pp. 267$278,2017$.
[7] R. Ambur and S. Rinderknecht, "Unbalance detection in rotor systems with active bearings using self-sensing piezoelectric actuators," Mech. Syst. Signal Process., vol. 102, pp. 72-86, 2018.

[8] R. Ta, D. O. Bukowitz-kulka, and J. C. Ustiolaborjas, "Equilibrado dinámico," vol. 84, pp. 415-421, 2009 .

[9] S. V Amiouny, J. J. Bartholdi III, and J. H. Vande Vate, "Heuristics for Balancing Turbine Fans," Oper. Res., vol. 48, no. 4, pp. 591-602, 2000.

[10] M. Jung and S. Jung, "Neural Network Control for the Balancing Performance of a Single-Wheel Transportation Vehicle: Gyrocycle," Adv. Intell. Syst. Comput., vol. 274, pp. 877-885, 2014.

[11] S. Zhou and J. Shi, "Active balancing and vibration control of rotating machinery: A survey," Shock Vib. Dig., vol. 33, no. 5, pp. 361-371, 2001.

[12] W. C. Foiles, P. E. Allaire, and E. J. Gunter, "Review: Rotor balancing," Shock Vib., vol. 5, no. 5-6, pp. 325-336, 1998.

[13] S. Liu, "A modified low-speed balancing method for flexible rotors based on holospectrum," Mech. Syst. Signal Process., vol. 21, no. 1, pp. 348-364, 2007.

[14] A. Mohammadzadeh and A. Ghoddoosian, "Balancing of flexible rotors with optimization methods," Int. Rev. Mech. Eng., vol. 4, no. 7, pp. 917923, 2010.

[15] L. Alberto et al., "An improvement of a singleplane balancing method via residual current of a rotor directly coupled to an induction motor," Nov. Sci., vol. 8, no. 16, pp. 59-76, 2016.

[16] P. Diouf and W. Herbert, "Understanding rotor balance for electric motors," IEEE Conf. Rec. Annu. Pulp Pap. Ind. Tech. Conf., pp. 7-17, 2014.

[17] Q. Wang and F. Wang, "A new vibration mechanism of balancing machine for satellite-borne spinning rotors," Chinese J. Aeronaut., vol. 27, no. 5, pp. 1318-1326, 2014.

[18] A. G. Kol'tsov and D. S. Rechenko, "Dynamic rotary balancing in metal-cutting machines," Russ. Eng. Res., vol. 32, no. 2, pp. 179-181, 2012.

[19] Š. Olejárová and T. Krenický, "Measuring the 
Size of Vibrations on a Mill Using the Vibration Analysis," Key Eng. Mater., vol. 756, pp. 119-126, 2017.

[20] M. M. Tahir, A. Hussain, S. Badshah, A. Q. Khan, and N. Iqbal, "Classification of unbalance and misalignment faults in rotor using multi-axis time domain features," ICET 2016 - 2016 Int. Conf. Emerg. Technol., 2017. 